VOL. $64(2001)$ [515-526]

\title{
POISSON MANIFOLDS IN GENERALISED HAMILTONIAN BIOMECHANICS
}

\author{
V. Ivancevic and C.E.M. Pearce
}

\begin{abstract}
In this paper the generalised Hamiltonian approach to the modelling of dynamical systems is developed not via the standard formalism of symplectic geometry but rather via Poisson manifolds and evolution equations. This alternative approach has the merit of being available in a wider context than the former. Application is made to three biomechanical models, one in which the symplectic-geometry approach also applies (the motion of a body segment) and two in which it does not (Schwan's model of blood and lymph circulation and Davydov's molecular model of muscle contraction).
\end{abstract}

\section{INTRODUCTION}

The classical development of Hamiltonian formalism in physical applications is usually based on symplectic geometry. This approach is well-defined in the finitedimensional case. In this case the algorithm has three steps, which we outline in Section 2. We then dwell briefly on the limitations on this approach and present an alternative method whereby they may be overcome. These ideas appear to hold considerable potential for applications to biomechanics, and it is our hope that we may draw the attention of readers in mathematical biology to the existence of tools which have been to date almost exclusively the preserve of the mathematical physicist. To this end we also present briefly three illustrative examples from biomechanics.

A quite elaborate machinery has been developed with diverse and extensive applications in mathematical physics and it is impossible to do this material justice within the confines of a short general article such as the present one. We can hope only to give some flavour, motivation and references. For further detail, we refer the reader in particular to the beautiful treatments of Arnold [3, Chapter 8], Abraham and Marsden [1, Chapter 3] and Abraham, Marsden and Ratiu [2, Chapter 8]. We note several other key references during our discussion.

Received Received by the Bulletin 10th January, 2001. Submitted to the Journal of the Australian Mathematical Society Series B 12th September, 1997, and subsequently transferred to the Bulletin.

Copyright Clearance Centre, Inc. Serial-fee code: 0004-9727/01 \$A2.00+0.00. 


\section{Symplectic Geometry}

We now proceed to the three-stage symplectic-geometry algorithm alluded to in the introduction.

A set $S$ is given the structure of a differentiable manifold if it is provided with a finite or countable collection of charts so that every point is represented in at least one chart. A chart is an open set $U$ in a coordinate space together with a one-to-one mapping $\psi: U \rightarrow S$. We assume that if two points $p, p^{\prime}$ in two charts $U, U^{\prime}$ have the same image in $S$, then $p$ and $p^{\prime}$ have neighbourhoods $V \subset U$ and $V^{\prime} \subset U^{\prime}$ with the same image in $S$. In this way we get a mapping $\psi^{\prime-1} \psi: V \rightarrow V^{\prime}$. The charts $U, U^{\prime}$ are termed compatible if such functions are differentiable. An atlas is a union of compatible charts. Two atlases are equivalent if their union is also an atlas.

A $C^{\infty}$-differentiable structure $\mathcal{D}$ on a space $S$ represents a class of equivalent atlases on $S$. A pair $M=(S, \mathcal{D})$ is termed a smooth manifold. If a covering by charts takes their values in a Banach space $E$, then $E$ is called the model space and $M$ is referred to as a $C^{\infty}$ Banach manifold modelled on $E$. A smooth manifold $M$ is called compact if every covering of $M$ by open charts $U_{\alpha}$ possesses a finite subcollection $U_{\alpha_{i}}$ also covering $M$. A smooth manifold $M$ is called Hausdorff (or separable) if any two of its distinct points have disjoint neighbourhoods.

Suppose $f: M_{1} \rightarrow M_{2}$, where $M_{1}$ and $M_{2}$ are smooth manifolds. The map $f$ is called a $C^{\infty}$-diffeomorphism if it is a bijection and both $f$ and $f^{-1}: M_{2} \rightarrow M_{1}$ are of class $C^{\infty}$. In this event $M_{1}$ and $M_{2}$ are termed diffeomorphic.

Denote by $T_{x} M$ the tangent space to a smooth manifold $\mathrm{M}$ at the point $x$. The tangent bundle $T M$ represents a union $\bigcup_{x \in M} T_{x} M$, together with the standard topology on $T M$ and a natural smooth manifold structure, the dimension of which is twice the dimension of the smooth manifold $M$ (see Steenrod [11]). (For each chart $(U, \phi)$ on $M$, given $x \in U$ and a $C^{\infty}$ canonical projection $\pi: T M \rightarrow M, \pi(v)=x$, we define a bijection $T \phi: \pi^{-1}(U) \rightarrow \phi(U) \times \mathbf{R}^{\mathbf{n}}$ by $T \phi(v)=\left(\phi(x), d_{x} \phi(v)\right)$ for $v \in T_{x} M$ and the linear isomorphism $d_{x} \phi: T_{x} M \rightarrow \mathbf{R}^{\mathbf{n}}$. The standard topology on $T M$ is defined by requiring any region $W \subset T M$ to be open if and only if the set $T \phi\left(W \cap \pi^{-1}(U)\right)$ is open in $\phi(U) \times \mathbf{R}^{\mathbf{n}}$.) A vector field $X$ on $M$ represents a section $X: M \rightarrow T M$ of the tangent bundle $T M$. Suppose $X(x) \in T_{x} M$ is a $C^{\infty}$-vector field on $M$. If $M$ is Hausdorff, existence and uniqueness conditions are satisfied sufficient for there to be a one-parameter group of diffeomorphisms or flow $\phi_{t}: M \rightarrow M$ given by $\left.\frac{d}{d t}\right|_{t=0} \phi_{t} x=X(x)$. We refer to $X$ as the 'velocity vector field' of the flow.

Analogously let $T_{x}^{*} M$ be the cotangent space to $M$ at $x$, the dual to its tangent space $T_{x} M$. The cotangent bundle $T^{*} M$ represents a union $\bigcup_{x \in M} T_{x}^{*} M$, together with the standard topology on $T^{*} M$ and a natural smooth manifold structure, the dimension of which is twice the dimension of $M$ (see Steenrod [11]). (For each chart $(U, \phi)$ on $M$, 
given $x \in U$ and a $C^{\infty}$ canonical projection $\pi^{*}: T^{*} M \rightarrow M, \pi^{*}(\alpha)=x$, we define a bijection $(T \phi)_{*}: \pi^{*-1}(U) \rightarrow \phi(U) \times\left(\mathbf{R}^{\mathrm{n}}\right)^{*}$ by $(T \phi)_{*}(\alpha)=\left(\phi \circ \pi^{*}(\alpha),\left(d_{x} \phi\right)_{*}(\alpha)\right)$ for $\alpha \in T_{x}^{*} M$ and the linear isomorphism $\left(d_{x} \phi\right)_{*}: T_{x}^{*} M \rightarrow\left(\mathbf{R}^{\mathbf{n}}\right)^{*}$. The standard topology on $T^{*} M$ is defined by the requirement that $W$ be open in $T^{*} M$ if and only if, for each chart $(U, \phi)$ on $M$, the set $(T \phi)_{*}\left(W \cap \pi^{*-1}(U)\right)$ is open in $\phi(U) \times\left(\mathbf{R}^{\mathbf{n}}\right)^{*}$.) A one-form $\theta$ on $M$ represents a section $\theta: M \rightarrow T^{*} M$ of the cotangent bundle $T^{*} M$.

Let $L^{2}(M, \mathbf{R})$ be the space of all bilinear maps $\omega: M \times M \rightarrow \mathbf{R}$ on the smooth manifold $M$. A bilinear map $\omega \in L^{2}(M, \mathbf{R})$ is nondegenerate if its matrix is nonsingular and skew-symmetric if its transpose $\omega^{t}$ equals $-\omega$. A skew-symmetric bilinear map $\omega$ is nondegenerate if and only if the manifold $M$ has even dimension, $2 n$ say.

Let $A^{2}(M, \mathbf{R})$ be the space of all skew-symmetric bilinear maps on a smooth manifold $M$. Then $\omega \in A^{2}(M, \mathbf{R})$ is called a two-form on $M$ and is said to be closed if $d \omega=0$, where $d$ denotes exterior derivative.

A symplectic form or symplectic structure on a smooth manifold $M$ is a closed twoform $\omega$ on $M$ for which $\omega(x)$ is nondegenerate for each $x \in M$.

Suppose $Q$ represents an $n$-dimensional configuration manifold of a mechanical system, with $Q$ modelled on a Banach space $E$. Then the phase space of the system is a $2 n$-dimensional manifold $P$ representing the cotangent bundle $T^{*} Q$ of the configuration manifold $Q$.

Suppose $U$ is open on the phase-space manifold $P$. A nondegenerate symplectic two-form $\omega$ on $P$ can be defined in local coordinates $q^{i}, p_{i} \in U$ by $\omega=d q^{i} \wedge d p_{i}$. Here ' $\wedge$ ' denotes the wedge or exterior product and as elsewhere in the text summation over $i$ is understood. The coordinates $q^{i}, p_{i} \in U$ are termed canonical. The usual procedure is for the canonical one-form $\theta$ to be defined first by $\theta=p_{i} d q^{i}$ and the canonical two-form $\omega$ then to be defined by $\omega=-d \theta$.

A symplectic phase-space manifold is a pair $(P, \omega)$.

The first ingredient of the symplectic geometry algorithm, Step A, is to find a symplectic phase-space manifold $(P, \omega)$.

Now suppose we have a symplectic manifold $(P, \omega)$. A vector field $X: P \rightarrow T P$ is called Hamiltonian if there is a smooth function $F: P \rightarrow \mathbf{R}$ such that the interior product or contraction $i_{X} \omega$ of the vector field $X$ and the two-form $\omega$ is equal to $d F$. We term $X$ locally Hamiltonian if $i_{X} \omega$ is closed.

Suppose a system has kinetic energy $T$ and potential energy $U^{*}$ and suppose the total energy $T+U^{*}$ is given by the smooth real-valued Hamiltonian function $H(q, p)$ in local canonical coordinates $q^{i}, p_{i}$. The Hamiltonian vector field $X_{H}$, determined by the condition $i_{X_{H}} \omega=d H$, is in a local chart $U$ defined as

$$
X_{H}=\left(\frac{\partial H}{\partial p_{i}},-\frac{\partial H}{\partial q^{i}}\right)=\frac{\partial H}{\partial p_{i}} \frac{\partial}{\partial q^{i}}-\frac{\partial H}{\partial q^{i}} \frac{\partial}{\partial p_{i}} .
$$

If $I$ denotes the $n \times n$ identity matrix and $\nabla$ the gradient operator, this equation can be 
represented compactly as $X_{H}=J \nabla H$, where $J=\left(\begin{array}{cc}0 & I \\ -I & 0\end{array}\right)$.

The second component of the algorithm, Step B, is to find a Hamiltonian vector field $X_{H}$ on $(P, \omega)$.

Now we have a symplectic phase-space manifold $(P, \omega)$ and a Hamiltonian vector field $X_{H}=J \nabla H$ corresponding to a smooth real-valued Hamiltonian function $H$ : $P \rightarrow \mathbf{R}$. If a unique one-parameter group of diffeomorphisms $\phi_{t}: P \rightarrow P$ exists with $\left.\frac{d}{d t}\right|_{t=0} \phi_{t} x=J \nabla H(x)$, then it is called the Hamiltonian phase flow.

The final ingredient, Step $C$, is to find a phase flow $\phi_{t}$ of $X_{H}$.

A smooth curve $t \mapsto\left(q^{i}(t), p_{i}(t)\right)$ on $(P, \omega)$ represents an integral curve of the Hamiltonian vector field $X_{H}=J \nabla H$ if Hamilton's equations

$$
\dot{q}^{i}=\frac{\partial H}{\partial p_{i}}, \quad \dot{p}_{i}=-\frac{\partial H}{\partial q^{i}}
$$

hold in local canonical coordinates.

An integral curve is said to be maximal if it is not a restriction of an integral curve defined on a larger interval of $\mathbf{R}$. From a standard theorem on the existence and uniqueness of the solution of a system of ordinary differential equations with smooth right-hand sides, it follows that if the manifold $(P, \omega)$ is Hausdorff, then there exists a maximal integral curve of $X_{H}=J \nabla H$ passing through any point $x=\left(q^{i}, p_{i}\right)$ for $t=0$. If $X_{H}$ is complete, that is, $X_{H}$ is $C^{\infty}$ and $(P, \omega)$ compact, the maximal integral curve of $X_{H}$ is the Hamiltonian phase flow $\phi_{t}$.

Denote by $\phi_{t}^{*} \omega$ the pull-back of $\omega$ by $\phi_{t}$ and by $L_{X_{H}} \omega$ the Lie derivative of $\omega$ on $X_{H}$. Then the phase flow $\phi_{t}$ is symplectic if $\omega$ is constant along $\phi_{t}$, that is, $\phi_{t}^{*} \omega=\omega$, if and only if $L_{X_{H}} \omega=0$. Symplectic phase flow $\phi_{t}$ consists of canonical transformations on $(P, \omega)$, that is, diffeomorphisms in canonical coordinates $q^{i}, p_{i}$ which leave $\omega$ invariant. In this case we have the Liouville theorem, which states that $\phi_{t}$ preserves phase-volume on $(P, \omega)$. Also, the total energy $H$ of the system is conserved along $\phi_{t}$, that is, $H \circ \phi_{t}=\phi_{t}$ (see [1] and [3]).

Suppose $(P, \omega)$ is a symplectic phase-space manifold. For any two real-valued smooth functions $f, g: P \rightarrow \mathbf{R}$, the classical finite-dimensional Poisson bracket $\{f, g\}=\omega\left(X_{f}, X_{g}\right)$ on $(P, \omega)$ is given in local canonical coordinates $q^{i}, p_{i}$ by

$$
\{f, g\}=\frac{\partial f}{\partial p_{i}} \frac{\partial g}{\partial q^{i}}-\frac{\partial f}{\partial q^{i}} \frac{\partial g}{\partial p_{i}} .
$$

The functions $f$ and $g$ are said to be in involution if $\{f, g\}=0$.

In the Poisson-bracket formulation, the time evolution of any function $A\left(q^{i}, p_{i}\right)$ defined along the trajectories of a Hamiltonian system is given by $\dot{A}=[A, H]$. Further, we have Hamilton's equations

$$
\dot{q}^{i}=\left[q^{i}, H\right], \quad \dot{p}_{i}=\left[p_{i}, H\right] .
$$


In the case of infinite-dimensional Hamiltonian systems, there is a distinction between weak and strong symplectic manifolds, depending on the reflexivity of the Banach model space.

The symplectic-geometry approach is basically the same as in the finite-dimensional case. One finds the infinite-dimensional Banach manifold $Q^{\infty}$ (the configuration space of the system) and defines the smooth phase-space manifold $P^{\infty}$ as a cotangent bundle $T^{*} Q^{\infty}$ of $Q^{\infty}$. The canonical one-form $\theta$ on $P^{\infty}$ is defined by $\theta(\alpha) \beta=\alpha \cdot T \pi(\beta)$, where $\alpha \in T^{*} Q^{\infty}, \beta \in T_{\alpha}\left(T^{*} Q^{\infty}\right)$ and $\pi: T^{*} Q^{\infty} \rightarrow Q^{\infty}$ is a projection. The weakly nondegenerate symplectic two-form $\omega$ on $P^{\infty}$ is defined as $\omega=-d \theta$.

The symplectic form $\omega$ is strongly nondegenerate if $Q^{\infty}$ is a reflexive Banach manifold, that is, a smooth manifold modelled on the reflexive Banach space $E^{\infty}$ (see Marsden [9]). If $\omega$ is only weakly nondegenerate, then given a smooth Hamiltonian function $H: P^{\infty} \rightarrow$ $\mathrm{R}$, a Hamiltonian vector field $X_{H}$ determined by $i_{X_{H}} \omega=d H$ is only densely defined and need not exist on all of $P^{\infty}$. Even if there exists a unique flow $\phi_{t}: P^{\infty} \rightarrow P^{\infty}$, representing a solution of a partial differential equation, as it is weakly symplectic $\left(\phi_{t}^{*} \omega=\omega\right)$ and energy is densely conserved along $\phi_{t}\left(H \circ \phi_{t}=\phi_{t}\right)$, it need not exist on all of $P^{\infty}$ (see [2] and [9]).

Whilst there is a strong symplectic approach for Hamiltonian particles described by ordinary differential equations, there is only a weak symplectic approach for many important Hamiltonian fields described by partial differential equations.

This provides a motivation for the present paper. Instead of using symplectic structures arising in Hamiltonian biomechanics, we propose the more general Poisson manifold $\left(\mathrm{g}^{*},\{F, G\}\right)$. Here $\mathbf{g}^{*}$ is a chosen Lie algebra with a $( \pm)$ Lie-Poisson bracket $\{F, G\}_{ \pm}(\mu)$ and carries an abstract Poisson evolution equation $\dot{F}=\{F, H\}$. This approach is welldefined in both the finite- and the infinite-dimensional case. It is equivalent to the strong symplectic approach when this exists and offers a viable formulation for Poisson manifolds which are not symplectic.

We show how the basic structure of a variety of biomechanical systems can be uniquely described by the Poisson manifold and the abstract evolution equation on it. In the following section we outline the machinery of Poisson manifolds and in Section 4 provide several concrete examples.

\section{The Poisson Manifold}

Let $E_{1}$ and $E_{2}$ be Banach spaces. A continuous bilinear functional $\left\langle_{,}\right\rangle: E_{1} \times E_{2} \rightarrow \mathbf{R}$ is nondegenerate if $\langle x, y\rangle=0$ implies $x=0$ and $y=0$ for all $x \in E_{1}$ and $y \in E_{2}$. We say $E_{1}$ and $E_{2}$ are in duality if there is a nondegenerate bilinear functional $\langle\rangle:, E_{1} \times E_{2} \rightarrow \mathrm{R}$. This functional is also referred to as an $L^{2}$-pairing of $E_{1}$ with $E_{2}$.

A Lie algebra consists of a vector space $g$ (usually a Banach space) carrying a bilinear skew-symmetric operation [,] $: \mathbf{g} \times \mathbf{g} \rightarrow \mathrm{g}$, called the commutator or Lie bracket. This 
represents a pairing $[\xi, \eta]=\xi \eta-\eta \xi$ of elements $\xi, \eta \in \mathrm{g}$ and satisfies Jacobi's identity

$$
[[\xi, \eta], \mu]+[[\eta, \mu], \xi]+[[\mu, \xi], \eta]=0 .
$$

Let $\mathbf{g}$ be a (finite- or infinite-dimensional) Lie algebra and $\mathbf{g}^{*}$ its dual Lie algebra, that is, the vector space $L^{2}$ paired with $\mathbf{g}$ via the inner product $\langle\rangle:, \mathbf{g}^{*} \times \mathbf{g} \rightarrow \mathbf{R}$. If $\mathbf{g}$ is finite-dimensional, this pairing reduces to the usual action (interior product) of forms on vectors. The standard way of describing any finite-dimensional Lie algebra $\mathbf{g}$ is to provide its $n^{3}$ structural constants $c_{i j}^{k}$, defined by $\left[\xi_{i}, \xi_{j}\right]=c_{i j}^{k} \xi_{k}$, in some basis $\xi_{i},(i=1, \ldots, n)$.

For any two smooth functions $F: \mathbf{g}^{*} \rightarrow \mathbf{R}$, we define the Fréchet derivative $D$ on the space $L\left(\mathbf{g}^{*}, \mathbf{R}\right)$ of all linear diffeomorphisms from $\mathbf{g}^{*}$ to $\mathbf{R}$ as a map $D F: \mathbf{g}^{*} \rightarrow$ $L\left(\mathbf{g}^{*}, \mathbf{R}\right) ; \mu \mapsto D F(\mu)$. Further, we define the functional derivative $\delta F / \delta \mu \in \mathbf{g}$ by

$$
D F(\mu) \cdot \delta \mu=\left\langle\delta \mu, \frac{\delta F}{\delta \mu}\right\rangle
$$

with arbitrary 'variations' $\delta \mu \in \mathrm{g}^{*}$.

For any two smooth functions $F, G: \mathbf{g}^{*} \rightarrow \mathbf{R}$, we define the $( \pm)$ Lie-Poisson bracket by

$$
\{F, G\}_{ \pm}(\mu)= \pm\left\langle\mu,\left[\frac{\delta F}{\delta \mu}, \frac{\delta G}{\delta \mu}\right]\right\rangle
$$

(see Weinstein [13] and Abraham, Marsden and Ratiu [2]). Here $\mu \in \mathbf{g}^{*},[\xi, \mu]$ is the Lie bracket in $\mathbf{g}$ and $\delta F / \delta \mu, \delta G / \delta \mu \in \mathbf{g}$ are the functional derivatives of $F$ and $G$.

The $( \pm$ ) Lie-Poisson bracket (3.1) is clearly a bilinear and skew-symmetric operation. It alșo satisfies the Jacobi identity

$$
\{\{F, G\}, H\}_{ \pm}(\mu)+\{\{G, H\}, F\}_{ \pm}(\mu)+\{\{H, F\}, G\}_{ \pm}(\mu)=0,
$$

thus confirming that $\mathbf{g}^{*}$ is a Lie algebra, as well as Leibniz' rule

$$
\{F G, H\}_{ \pm}(\mu)=F\{G, H\}_{ \pm}(\mu)+G\{F, H\}_{ \pm}(\mu) .
$$

(see [2] and [13]).

If $\mathbf{g}$ is a finite-dimensional phase-space manifold with structure constants $c_{i j}^{k}$, the $( \pm)$ Lie-Poisson bracket becomes

$$
\{F, G\}_{ \pm}(\mu)= \pm \mu_{k} c_{i j}^{k} \frac{\delta F}{\delta \mu_{i}} \frac{\delta G}{\delta \mu_{j}} .
$$

The $( \pm)$ Lie-Poisson bracket represents a Lie-algebra generalisation of the classical finite-dimensional Poisson bracket $[F, G]=\omega\left(X_{f}, X_{g}\right)$ on the symplectic phase-space manifold $(P, \omega)$ for any two real-valued smooth functions $F, G: P \rightarrow \mathbf{R}$. 
As in the classical case, any two smooth functions $F, G: \mathbf{g}^{*} \rightarrow \mathbf{R}$ are in involution if $\{F, G\}_{ \pm}(\mu)=0$.

The Lie-Poisson theorem states that a Lie algebra $g^{*}$ with a \pm Lie-Poisson bracket $\{F, G\}_{ \pm}(\mu)$ represents a Poisson manifold $\left(\mathbf{g}^{*},\{F, G\}_{ \pm}(\mu)\right)$ (see $\left.[2,3,13]\right)$.

Given a smooth Hamiltonian function $H: \mathbf{g}^{*} \rightarrow \mathbf{R}$ on the Poisson manifold $\left(\mathbf{g}^{*},\{F, G\}_{ \pm}(\mu)\right)$, the time evolution of any smooth function $F: \mathbf{g}^{*} \rightarrow \mathbf{R}$ is given by the abstract Poisson evolution equation

$$
\dot{F}=\{F, H\}
$$

$($ see $[2,3,13])$.

\section{BIOMECHANICAL EXAMPLES}

4.1. Three-axial joint motion A finite-dimensional example of the Poisson manifold and evolution equation is taken from the macroscopic biomechanical level of human body motion. The dynamics of human body-segments (see Hatze [7] and Ivancevic [8] for a classical, Lagrangian approach) may be also prescribed by Euler's equations of rigid body dynamics. The equations of motion for a free rigid body, described by an observer fixed on the moving body, are usually given by Euler's vector equation

$$
\dot{\Pi}=\Pi \times w .
$$

Here $\Pi, w \in \mathbf{R}^{3}, \Pi_{i}=I_{i} w_{i}$ and $I_{i}(i=1,2,3)$ are the principal moments of inertia, the coordinate system in the segment is chosen so that the axes are principal axes, $w$ is the angular velocity of the body and $\Pi$ is the angular momentum of the segment.

The kinetic energy of the segment is the Hamiltonian function $H: \mathbf{R}^{3} \rightarrow \mathbf{R}$ given by

$$
H(\Pi)=\frac{1}{2} \Pi \cdot w
$$

and is a conserved quantity for (4.1).

The vector space $\mathbf{R}^{3}$ is a Lie algebra with respect to the bracket operation given by the usual cross product. The space $\mathbf{R}^{3}$ is paired with itself via the usual dot product. So if $F: \mathbf{R}^{3} \rightarrow \mathbf{R}$, then $\delta F / \delta \Pi=\nabla F(\Pi)$ and the (-) Lie-Poisson bracket $\{F, G\}_{-}(\Pi)$ is given via (3.1) by the triple product

$$
\{F, G\}_{-}(\Pi)=-\Pi \cdot(\nabla F(\Pi) \times \nabla G(\Pi)) .
$$

Euler's vector equation (4.1) represents a generalised Hamiltonian system in $\mathbf{R}^{3}$ relative to the Hamiltonian function $H(\Pi)$ and the (-) Lie-Poisson bracket $\{F, G\}_{-}(\Pi)$. Thus the Poisson manifold $\left(\mathrm{R}^{3},\{F, G\}_{-}(\Pi)\right)$ is defined and the abstract Poisson equation is equivalent to Euler's equation (4.1) for a body segment and associated joint. 
4.2. Hemodynamics An infinite-dimensional example of the Poisson manifold and evolution equation is provided by a macroscopic biomechanical model of hemodynamics. Equations for blood and lymph circulation have been usually given based on Euler's equations for ideal fluids (see Schwan [10] and Ivancevic [8]). The equations of motion for an ideal incompressible homogenous fluid in a region $\Omega \in \mathbf{R}^{3}$ with smooth boundary $\partial \Omega$ are usually prescribed by Euler's vector equation

$$
\frac{\partial \mathbf{v}}{\partial t}+(\mathbf{v} \cdot \nabla) \mathbf{v}=-\nabla \mathbf{p}
$$

where $\mathbf{v}(\mathbf{t}, \mathbf{x}) \in \mathrm{T}_{\mathbf{x}}(\partial \Omega)$ for $\mathbf{x} \in \partial \Omega$ is the Eulerian or spacial velocity, a time-dependent vector field on $\Omega$. This field is divergence-free $(\nabla \cdot v=0)$ with initial condition $v(0, x)=$ $\mathbf{v}_{\mathbf{0}}(\mathbf{x})$. The pressure $p$ is a function of $\mathbf{v}$ and is determined up to a constant by $\mathbf{v}$ via the Neumann problem (see [2] and [3]).

The kinetic energy of the fluid is

$$
H(\mathbf{v})=\frac{1}{2} \int_{\Omega}\|\mathbf{v}\|^{2} \mathbf{d}^{3} \mathbf{x}
$$

where $\|\cdot\| \Omega \rightarrow R, \mathbf{v} \mapsto\|\mathbf{v}\|$ denotes a norm on $\Omega$ and is a conserved quantity for (4.2) (see [3]).

The vector space $\chi_{\text {div }}(\Omega)$ of divergence-free vector fields on $\Omega$ tangent to $\partial \Omega$ is the Lie algebra with the bracket given by

$$
[\mathbf{u}, \mathbf{v}]=(\mathbf{v} \cdot \nabla) \mathbf{u}-(\mathbf{u} \cdot \nabla) \mathbf{v} .
$$

The $L^{2}$-pairing of $\chi_{\text {div }}(\Omega)$ with itself gives the functional derivative $\delta F / \delta \mathbf{v}$ for any smooth function $F: \chi_{\text {div }}(\Omega) \rightarrow \mathbf{R}$. In particular, $\delta H / \delta \mathbf{v}=\mathbf{v}$. The $(+)$ Lie-Poisson bracket on $\chi_{\text {div }}(\Omega)$ is via $(3.1)$ given by

$$
\{F, G\}_{+}(\mathbf{v})=\int_{\Omega} \mathbf{v} \cdot\left[\left(\frac{\delta \mathrm{G}}{\delta \mathbf{v}} \cdot \mathbf{v}\right) \frac{\delta \mathbf{F}}{\delta \mathbf{v}}-\left(\frac{\delta \mathbf{F}}{\delta \mathbf{v}} \cdot \mathbf{v}\right) \frac{\delta \mathrm{G}}{\delta \mathbf{v}}\right] \mathrm{d}^{3} \mathbf{x}
$$

(see [2] and [3]).

Euler's vector equation (4.2) for an ideal incompressible homogenous fluid is a Hamiltonian system on the Lie algebra $\chi_{\text {div }}(\Omega)$ relative to the $(+)$ Lie-Poisson bracket $\{F, G\}_{(+)}(\mathbf{v})$ and the Hamiltonian function $H(\mathbf{v})$ (see [2] and [3]). Therefore the Poisson manifold $\left(\chi_{\text {div }}(\Omega),\{F, G\}_{+}(v)\right)$ is defined and the abstract Poisson evolution equation $\dot{F}=\{F, H\}$, which holds for any smooth function $F: \chi_{\text {div }}(\Omega) \rightarrow \mathbf{R}$, is equivalent to Euler's equation (4.2).

4.3. Muscular contraction A second infinite-dimensional example of the Poisson manifold and evolution equation is taken from a model of muscular contraction at a microscopic level. The basis of the molecular model of muscular contraction is oscillations of 
Amid I peptide groups with associated dipole electric momentum inside a spiral structure of myosin filament molecules. The reader is referred to Davydov [4] and Ivancevic [8].

There is a simultaneous resonant interaction and strain interaction generating a collective interaction directed along the axis of the spiral. The resonance excitation jumping from one peptide group to another can be represented as an exciton, the local molecule strain caused by the static effect of excitation as a phonon and the resultant collective interaction as a soliton.

The simplest model, of Davydov's solitary particle-waves, is given by the nonlinear Schrödinger equation

$$
i \frac{\partial \psi}{\partial t}=-\frac{\partial^{2} \psi}{\partial x^{2}}+2 \chi|\psi|^{2} \psi
$$

for $-\infty<x<+\infty$. Here $\psi(x, t)$ is a smooth complex-valued wave function with initial condition $\left.\psi(x, t)\right|_{t=0}=\psi(x)$ and $\chi$ is a nonlinear parameter. In the linear limit $(\chi=0)$ (4.3) becomes the ordinary Schrödinger equation for the wave function of the free onedimensional particle with mass $m=1 / 2$.

We may define the infinite-dimensional phase-space manifold $\mathcal{P}=\{(\psi, \bar{\psi}) \in$ $S(\mathbf{R}, \mathbf{C})\}$, where $S(\mathbf{R}, \mathbf{C})$ is the Schwartz space of rapidly-decreasing complex-valued functions defined on $\mathbf{R}$. We define also the algebra $\chi(\mathcal{P})$ of observables on $\mathcal{P}$ consisting of real-analytic functional derivatives $\delta F / \delta \psi, \delta F / \delta \bar{\psi} \in S(\mathbf{R}, \mathbf{C})$.

The Hamiltonian function $H: \mathcal{P} \rightarrow \mathbf{R}$ is given by

$$
H(\psi)=\int_{-\infty}^{+\infty}\left(\left|\frac{\partial \psi}{\partial x}\right|^{2}+\chi|\psi|^{4}\right) d x
$$

and is equal to the total energy of the soliton. It is a conserved quantity for (4.3) (see Takhtajan and Fadeev [12]).

The Poisson bracket on $\chi(\mathcal{P})$ represents a direct generalisation of the classical finitedimensional Poisson bracket

$$
\{F, G\}_{+}(\psi)=i \int_{-\infty}^{+\infty}\left(\frac{\delta F}{\delta \psi} \frac{\delta G}{\delta \bar{\psi}}-\frac{\delta F}{\delta \bar{\psi}} \frac{\delta G}{\delta \psi}\right) d x
$$

It manifestly exhibits skew-symmetry and satisfies Jacobi's identity. The functionals are given by $\delta F / \delta \psi=-i\{F, \bar{\psi}\}$ and $\delta F / \delta \bar{\psi}=i\{F, \psi\}$. Therefore the algebra of observables $\chi(\mathcal{P})$ represents the Lie algebra and the Poisson bracket is the $(+)$ Lie-Poisson bracket $\{F, G\}_{+}(\psi)$.

The nonlinear Schrödinger equation (4.3) for the solitary particle-wave is a Hamiltonian system on the Lie algebra $\chi(\mathcal{P})$ relative to the $(+)$ Lie-Poisson bracket $\{F, G\}_{+}(\psi)$ and Hamiltonian function $H(\psi)$ (see [12]). Therefore the Poisson manifold $(\chi(\mathcal{P})$, $\left.\{\mathrm{F}, \mathrm{G}\}_{+}(\psi)\right)$ is defined and the abstract Poisson evolution equation $\dot{F}=\{F, H\}$, which holds for any smooth function $F: \chi(\mathcal{P}) \rightarrow \mathbf{R}$, is equivalent to equation (4.3). 
A more subtle model of soliton dynamics is provided by the Korteveg-de Vries equation

$$
f_{t}-6 f f_{x}+f_{x x x}=0,
$$

where $x \in \mathbf{R}$ and $f$ is a real-valued smooth function on $\mathbf{R}$. This equation is related to the ordinary Schrödinger equation by the inverse scattering method. See Gardner et al. [6].

We may define the infinite-dimensional phase-space manifold $\mathcal{V}=\{f \in S(\mathrm{R})\}$, where $S(\mathbf{R})$ is the Schwartz space of rapidly-decreasing real-valued functions $\mathbf{R}$. 'We define further $\chi(\mathcal{V})$ to be the algebra of observables consisting of functional derivatives $\delta F / \delta f \in S(\mathbf{R})$.

The Hamiltonian $H: \mathcal{V} \rightarrow \mathbf{R}$ is given by

$$
H(f)=\int_{-\infty}^{+\infty}\left(f^{3}+\frac{1}{2} f_{x}^{2}\right) d x
$$

and provides the total energy of the soliton. It is a conserved quantity for (4.5) (see [3] and [6]).

As a real-valued analogue to (3.4), the $(+)$ Lie-Poisson bracket on $\chi(\mathcal{V})$ is given via (3.1) by

$$
\{F, G\}_{+}(f)=\int_{-\infty}^{+\infty} \cdot \frac{\delta F}{\delta f} \frac{d}{d x} \frac{\delta G}{\delta f} d x
$$

Again it possesses skew-symmetry and satisfies Jacobi's identity (see [6]). The functionals are given by $\delta F / \delta f=\{F, f\}$.

The Korteveg-de Vries equation (4.5), describing the behaviour of the molecular solitary particle-wave, is a Hamiltonian system on the Lie algebra $\chi(\mathcal{V})$ relative to the $(+)$ Lie-Poisson bracket $\{F, G\}_{+}(f)$ and the Hamiltonian function $H(f)$ (see [6]). Therefore the Poisson manifold $\left(\chi(\mathcal{V}),\{F, G\}_{+}(f)\right)$ is defined and the abstract Poisson evolution equation $\dot{F}=\{F, H\}$, which holds for any smooth function $F: \chi(\mathcal{V}) \rightarrow \mathbf{R}$, is equivalent to (4.5).

Finally, it is clear that the two solitary equations (4.3), (4.5) have a quantummechanical derivation. By the use of the first quantisation method, every classical biophysical observable $F$ is represented in the Hilbert space $L^{2}(\psi)$ of square-integrable complex psi-functions by a Hermitian (self-adjoint) linear operator $\widehat{F}$ with real eigenvalues. The classical Poisson bracket $\{F, G\}=K$ corresponds to the commutator $[\widehat{F}, \widehat{G}]=i \hbar \widehat{K}$. Therefore the classical evolution equation $\dot{F}=\{F, H\}$ corresponds, in the Heisenberg picture, to the quantum evolution equation $i \hat{\hbar} \widehat{\dot{F}}=[\widehat{F}, \widehat{H}]$ for any representative operator $\widehat{F}$ and quantum Hamiltonian $\widehat{H}$. By Ehrenfest's theorem, this equation is also valid for expectation values $\langle\cdot\rangle$ of observables, that is, $i \hbar\langle\hat{\dot{F}}\rangle=\langle[\widehat{F}, \widehat{H}]\rangle$ (see Dirac [5]). 


\section{ConClusion}

The Hamiltonian formalism in biophysical applications is usually based on symplectic geometry. This approach is well-defined in the finite-dimensional case and represented by a three-step algorithm. First, the phase-space manifold $(P, \omega)$ of the system is defined including the canonical coordinates. Then a Hamiltonian vector field $X_{H}$ on $(P, \omega)$ is derived from the Hamiltonian total energy function $H$. Finally the phase flow $\phi_{t}$ of $X_{H}$ is obtained as a solution of canonical Hamiltonian equations for given initial conditions.

In the case of infinite-dimensional Hamiltonian systems, the symplectic geometry approach is not well-defined for many important examples. In this case there is a distinction between weak and strong symplectic manifolds, depending on the reflexivity of the Banach model space. Unfortunately many biophysical systems have only weak symplectic structure.

In this paper the unique, generalised Hamiltonian approach to modelling is developed in the form of Poisson manifolds and evolution equations. As opposed to the standard symplectic approach, the generalised method of Poisson manifolds works well in both finite- and infinite-dimensional cases. This may be in the context of models which are physically microscopic or macroscopic. Applications of biomechanical systems presented include the basic models of biomechanical particles, fields, and particle-fields. We consider the rigid-body dynamics of human motion, fluid dynamics of blood and lymph circulation and the soliton dynamics of muscular contraction. The last-mentioned involves a nonlinear Schrödinger equation and Korteveg-de Vries equation derived from quantum mechanics. All have been shown to represent the same abstract evolution equation $\dot{F}=\{F, H\}$ on an appropriate Poisson manifold.

\section{REFERENCES}

[1] R. Abraham and J. Marsden, Foundations of mechanics (Addison-Wesley, Reading MA, 1978).

[2] R. Abraham, J. Marsden and T. Ratiu, Manifolds, tensor analysis and applications, Applied Mathematical Sciences 75 (Springer-Verlag, Berlin, Heidelberg, New York, 1988).

[3] V.I. Arnold, Mathematical methods of classical mechanics, Graduate Texts in Mathematics 60 (Springer-Verlag, Berlin, Heidelberg, New York, 1978).

[4] A.S. Davydov, Quantum mechanics (Pergamon Press, Oxford, 1976).

[5] P.A.M. Dirac, The principles of quantum mechanics (Oxford University Press, Oxford, 1967).

[6] C.S. Gardner, J.M. Greene, M.D. Kruskal and R.M. Muirat, 'Korteweg-de Vries equation and generalisations', Comm. Pure. Appl. Math. 27 (1974), 97-133.

[7] H. Hatze, 'A complete set of control equations for the human musculoskeletal system', $J$. Biomech. 10 (1977), 799-805.

[8] V. Ivancevic, Introduction to biomechanical systems: Modelling, control and learning (in Serbian) (Scientific Book, Belgrade, 1991). 
[9] J. Marsden, 'Generalised Hamiltonian mechanics', Arch. Rational Mech. Anal 28 (1968), 326-362.

[10] H.P. Schwan, Biological engineering (McGraw-Hill, New York, 1969).

[11] N. Steenrod, The topology of fibre bundles, Princeton Mathematical Series 14 (Princeton Univ. Press, Princeton, 1951).

[12] L.A. Takhtajan and L.D. Fadeev, Hamiltonian methods in the theory of solitons (in Russian), Springer series in Soviet Mathematics (Springer-Verlag, Berlin, Heidelberg, New York, 1986).

[13] A. Weinstein, 'The local structure of Poisson manifolds', J. Differential Geom. 18 (1983), 523-557.

Torson Productions Pty Ltd

Adelaide SA 5034

Australia
Applied Mathematics Department

The University of Adelaide

Adelaide SA 5005

Australia 